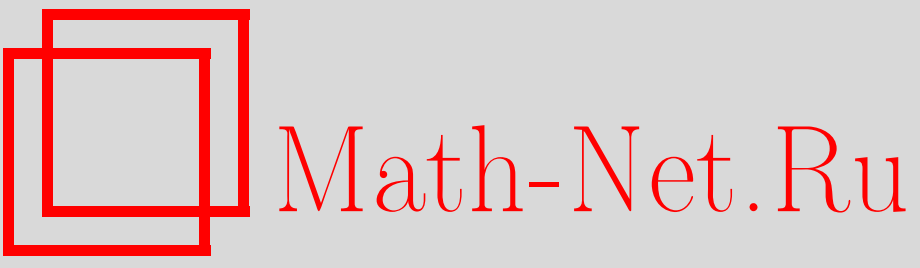

От правления Московского математического общества, УМН, 2004, том 59, выпуск $5,169-174$

DOI: https://doi.org/10.4213/rm788

Использование Общероссийского математического портала Math-Net.Ru подразумевает, что вы прочитали и согласны с пользовательским соглашением

http://www.mathnet.ru/rus/agreement

Параметры загрузки:

IP: 3.85 .183 .62

26 апреля 2023 г., 04:23:19 


\section{ОТ ПРАВЛЕНИЯ МОСКОВСКОГО МАТЕМАТИЧЕСКОГО ОБЩЕСТВА}

7 сентября 2004 г. состоялось Отчетно-выборное собрание Московского математического общества. Были заслушаны отчеты Правления и Ревизионной комиссии ММО. Работа Правления за отчетный период была признана удовлетворительной.

Состоялись выборы руководящих органов ММО.

Правление ММО избрано в составе: В.И. Арнольд - президент, В. А. ВАСИльЕВ, Ю. С. ИльяшЕнко - вице-президенты, С. П. Новиков - главный редактор журнала "Успехи математических наук", А.В. МихАЛЕв - казначей, Л.Р. ВоЛЕвич - главньй редактор трудов ММО, В.М. ТихомиРов - библиотекарь, В.М. БУХШтАБЕР - ответственный за краткие сообщения ММО и за контакты с Европейским математическим обществом, С. М. ГУСЕЙн-ЗАДЕ, И. А. ДынниКов - ученые секретари, А. М. АБРАмов - председатель секции средней школы, Д. В. АНОсов, А. В. БУЛИНСКИЙ, Э. Б. ВИНБЕРГ, В. М. ЗАКАЛЮКИН, М.И. ЗЕлИКИН, А.Г. КосТючЕНКО, И. М. КРИчеВеР, С.К. ЛАНДО, Р.А. МИНЛОС, А. И. НЕЙШТАДТ, А. Г. СЕРГЕЕВ, А. Б. СОСИНСКИЙ, Д. В. ТРЕШЕВ.

Ревизионная комиссия ММО избрана в составе: Ю.М. Смирнов (председатель), В. А. Голубева, А. Д. Мышкис, Е. Г. Скляренко, А. В. Фурсиков.

Были подведены итоги конкурса на премию ММО для молодых математиков. Лауреатом премии 2004 г. стал Т.Е. Панов за цикл работ "Инварианты многообразий с действиями групп".

Состоялись очередные заседания Московского математического общества.

Заседание 2 сентября 2003 г. В. И. Арнольд. Самые частые представления.

Насколько часто встречаются разные группы симметрий?

Унитарное представление конечной группы в эрмитовом пространстве назьвается частым, если размерность многообразия унитарно эквивалентных ему представлений (в том же пространстве) максимальна. Оказывается, кратности неприводимых составляющих частого представления пропорциональны их размерностям (по меньшей мере асимптотически, когда размерность эрмитова пространства велика), т.е. ситуация такая же, как в регулярном представлении в пространстве функций на группе. Такой же оказьвается ситуация с распределением собственных функций выдерживающего группу симметрий оператора (например, оператора Лапласа или Шрёдингера) по типам симметрий (автор уже формулировал эти результаты, но без доказательств, в работе "Моды и квазимоды" 1972 г. и в работах 1980-х годов по магнитной гидродинамике).

В случае, когда группа симметрий есть группа всех перестановок из $n$ элементов, максимальные составляющие частых представлений определяются специальньми диаграммами Юнга, например $(3+1+1,3+2+1,4+2+1,3+2+1+1)$, размерностей $(6,16,35,35)$, но удовлетворяют ли диаграммы Юнга представлений максимальной размерности асимптотическим законам Вершика форм болшших диаграмм Юнга перестановок и разложений больших чисел на множители, неизвестно.

Заседание 23 сентября 2003 г. А. Э. ГУтеРмАН, А. В. МихАЛЕВ. Линейные отображения, сохраняющие матричные инварианты.

Теория линейных отображений, сохраняющих матричные инварианты, восходит к классической работе Г. Фробениуса, в которой для построения теории представлений конечных групп потребовалось охарактеризовать линейные биективные отображения пространства матриц с комплексньми коэффициентами, не меняющие определитель. Впоследствии эта тематика получила 
свое развитие в работах Шура и Дьедонне. Особенно интенсивно указанное направление развивается в течение последних 30 лет, как в связи с целым рядом естественных алгебраических вопросов, так и в связи с различными приложениями. Современному состоянию теории посвящены специальные выпуски журнала "Linear and Multilinear Algebra": 1993 и 2001 годов.

В докладе дан исторический обзор основных результатов, отражено современное состояние рассматриваемой теории и ее взаимосвязь с другими областями современной математики. Приведены недавние результаты авторов в этом направлении. В том числе: некоммутативный вариант теоремы Фробениуса для определителя Дьёдонне и резултаты о сохранении супер-ранга матриц над градуированными алгебрами. Представлен новый метод решения указанных задач, который даже в случае матриц над полями позволяет получить ряд новых содержательных результатов, таких, как характеризация монотонных отображений, отображений, сохраняющих нули многочленов и серию важных отношений, определяемых в терминах ранга матриц. Представлена полуколцевая версия многих классических резултатов о линейных отображениях, сохраняющих матричные инварианты, и рассказано, что дает метод теории моделей для решения возникающих задач. Сформулирован ряд открытых вопросов.

Заседание 30 сентября 2003 г. А. Б. ВАСИЛЬЕВА, В.Ф. БУтУЗОВ, Н. Н. НЕфЕДОВ. Асимптотическая теория контрастных структур.

В докладе представлены полученные в последние годы резултаты авторов по исследованию решений с внутренними слоями в краевых задачах для нелинейных сингулярно возмущенных уравнений с частными производными. Такие решения назьваются контрастньпии структурами $(\mathrm{KC})$ и имеют широкие приложения в различных задачах математической физики. Даны ответы на следующие вопросы.

1. Существование контрастных структур.

2. Асимптотическое разложение контрастных структур по малому параметру.

3. Устойчивость контрастных структур.

4. Область влияния (притяжения) устойчивых контрастных структур.

Заседание 7 октября 2003 г. Студенческие чтения. Д. В. Аносов. От Ньютона к Кеплеру.

Речь идет о "Задаче Кеплера": материальная точка $P$ ("планета") притягивается к неподвижному центру $S$ ("солнце") обратно пропорционално квадрату расстояния. Вероятно, самое простое из многочисленных решений задачи Кеплера предложил У. Гамильтон. В докладе рассказано как на пути Гамильтона можно получить одновременную регуляризацию решений с фиксированной энергией, предложенную спустя 100 с лишним лет Ю. Мозером. При этом привлекается красивая геометрия в стиле середины XIX века.

Заседание 21 октября 2003 г. О.Н. АгеЕВ, А.А. ПРИходько, В. В. РЫжиков. О спектральных кратностях в эргодической теории. Проблема Рохлина.

Доклад посвящен проблеме исследования спектральных свойств динамических систем с инвариантной мерой. Классическая спектральная теорема для унитарного оператора в сепарабельном гильбертовом пространстве дает полную систему инвариантов оператора относительно унитарной эквивалентности: это - мера максимального спектралшного типа и функция кратности. Большой интерес представляет изучение спектральных инвариантов унитарных представлений, порожденных динамическими системами. Около 50 лет оставался открытым вопрос Рохлина о существовании эргодического преобразования с однородным спектром кратности 2. Было рассказано полученное в 1999 г. Агеевым и Рыжиковым решение данной проблемы.

Заседание 28 октября 2003 г. В. Л. ГинзБУрГ. Какие проблемы физики и астрофизики представляются особенно важными и интересными в начале XXI века?

Заседание 4 ноября 2003 г. В. В. Фок. Высшие пространства Тейхмюллера и кластерные алгебры.

Высшим пространством Тейхмюллера $\mathscr{X}(S, G)$ назьвается одна из связных компонент (выделяемая несложным условием положительности) пространства дискретных вложениий фундаментальной группы двумерной римановой поверхности в вещественную расщепимую простую группу Ли. В случае $G=P S L(2, \mathbb{R})$ это пространство, согласно теореме униформизации Пуанка- 
ре, совпадает с обычным пространством Тейхмюллера - пространством комплексных структур на $S$, рассматриваемых с точностью до дифффеоморфизмов, гомотопически эквивалентных тождественному. В случае $G=P S L(3, \mathbb{R})$ это пространство является пространством вьпуклых вещественно-проективных структур на $S$, введенных и изученных Голдманом (William Goldman) и Чоем (Suhyong Choi).

Понятие кластерной алгебры было введено Андреем Зелевинским и Сергеем Фоминым в связи с изучением систем алгебры функций на двойных клетках Брюа простых групп Ли. Слово “алгебра" в этом контексте не очень удачно, поскольку более естественно связьвать с этим понятием не алгебру, а категорию. Объекты этой категории задаются, с точностью до изоморфизма, кососимметризуемыми целочисленными матрицами, а морфизмы задаются явными образующими и соотношениями. У этой категории существует шесть замечательных реализаций в категории алгебр, (одна из которых и есть алгебра, служившая отправной точкой Зелевинскому и Фомину).

Основным резултатом, которьй изложен в докладе, является утверждение о том, что алгебра функций на обобщенных пространствах Тейхмюллера является реализацией кластерной алгебры. С одной стороны, это дает многие свойства общих кластерных алгебр, а с другой- насколько это возможно явное описание обобщенных пространств Тейхмюллера, действия на них модулярной группы, так назьваемого “тропического" или "ламинационного" предела, скобки Пуассона и квантования. Также удается проследить связь с кристальными базисами Касивары (Masaki Kashiwara) и Люстига (George Lustig), а также “сотовой” (honeycomb) конструкцией сплетающих операторов Кнутсона (Allen Knutson) и Тао (Terence Тао).

Доклад основан на совместной работе автора с А. Гончаровым.

Заседание 11 ноября 2003 г. Ю. А. НЕРЕТИн. Обратный предел унитарных групп, матричные интегралы типа Хуа Ло Кена и бесконечномерный аналог меры Хаара.

Рассматривается естественное выделенное отображение из большей унитарной группы $U(n+$ $m)$ в меньшую унитарную группу $U(n)$, оно переводит блочную матрицу $\left(\begin{array}{cc}A & B \\ C & D\end{array}\right)$ в матрицу $A-C(1+D)^{-1} B$ (эта формула обнаружена М. С. Лившицем в 1940 -х годах).

Рассматривается обратный предел пространств $U(n)$. Он не является группой, однако бесконечномерная унитарная группа действует на нем левыми и правыми умножениями. На этом обратном пределе строится естественное двухпараметрическое семейство вероятностных мер.

На построенном объекте существует гармонический анализ с большим количеством явных нетривиалшных формул. Дано неформальное введение в формулу Планшереля, полученную недавно А. М. Бородиньпм и Г. И. Ольшанским.

Заседание 25 ноября 2003 г. В.И. Арнольд. Динамика Ферма, арифметика матриц, конечная окружность и конечная плоскость Лобачевского.

Малая теорема Ферма имеет следующее матричное обобщение: если число $p$ простое, то разность между $p$-й степенью суммы нескольких слагаемых и суммой их $p$-х степеней делится на $p$ и даже представима в виде многочлена от основных симметрических функций слагаемых с делящимися на $p$ коэфофициентами.

Например, след $p$-й степени матрицы сравним со следом исходной матрицы по модулю $p$.

Конечная окружность состоит из пар вычетов по модулю $p$, сумма квадратов которых равна единице. Она является конечной коммутативной группой. Оказьвается, эта группа - циклическая, порядка либо $p+1$, либо $p-1$.

Описанные теории позволяют получить, например, такую теорему о динамической системе в пространстве унимодулярных матриц второго порядка из вычетов по простому модулю $p$ : цепочка, полученная из матрицы $k$ последовательными возведениями предыдущей матрицы цепочки в квадрат, заканчивающаяся единичной матрицей (предшествуемой не единичной) существует тогда и только тогда, когда число $p$ дает при делении на 2 в степени $k$ либо остаток 1 , либо остаток -1 .

Например, для $p=5$ и 11 максимальная длина $k$ цепочки равна 2 , а для $p=17$ и для $p=47$ максимальная длина $k$ равна 4 .

Заседание 16 декабря 2003 г. И. М. КрИЧЕВЕР, В. М. БУХшТАБЕР. Интегрируемые уравнения и геометрия Абелевых многообразий. 
Доказательство знаменитой гипотезы Новикова о том, что многообразия Якоби - это в точности главно-поляризованные абелевы многообразия, в тэта-функциях которых интегрируется уравнение Кадомцева-Петвиашвили, было получено в 1986 году Шиотой. Оно привело к решению одной из самых старых и знаменитых проблем алгебраической геометрии - проблемы Римана-Шоттки. Гипотеза Новикова была инспирована конструкцией алгебро-геометрических решений солитонных уравнений, предложенной одним из авторов доклада. Центральную роль в этой конструкции играло понятие функций Бейкера-Ахиезера, аналитические свойства которых на соответствующей вспомогательной алгебраической кривой гарантировали то, что эта функция удовлетворяет переопределенной системе линейных дифференциальных уравнений.

В 1995 году авторами доклада было обнаружено, что функции Бейкера-Ахиезера удовлетворяют функциональныц м уравнениям, имеющим вид теорем сложения. Эти уравнения содержат $g+1$ слагаемое, в отличие от классических теорем сложения для абелевых тэта-функций, содержащих $2^{g}+1$ слагаемое. Это принципиальное различие привело авторов к гипотезе о том, что полученные формулы сложения являются храктеристическими для многообразий Якоби. Недавно эта гипотеза была доказано С. Грушевским, который показал также, что следствием предложенных нами формул сложения является знаменитая формула тройных секущих Фэя и ее далекие обобщения, предложенные Ганнингом.

Основной целью доклада является обзор этих и ряда других смежных резултатов, относящихся, в частности, к нерешенной до сих пор проблеме характеризации многообразий Прима.

Заседание 17 февраля 2004 г. А. В. Болсинов. Интегрируемые геодезические потоки на римановых многообразиях: конструкции и препятствия.

Геодезический поток - это система дифференциальных уравнений, описывающая движение точки по римановому многообразию (например, по поверхности в трехмерном пространстве) в отсутствие внешних сил. Интегрируемость геодезического потока можно понимать двояко. С аналитической точки зрения она означает возможность решения системы в квадратурах, с топологической - регулярность глобального поведения интегралшных траекторий. Хорошо известно, что интегрируемость - явление исключительное. Разумеется, нет никакой проблемы построить локальные примеры интегрируемых геодезических потоков. Однако в целом на компактном многообразии сделать это можно далеко не всегда. Основной вопрос, которьй обсуждается в докладе, формулируется так: какие замкнутые многообразия допускают интегрируемые геодезические потоки? Недавно было обнаружено, что ответ зависит от функционального класса, с которым мы хотим работать. Например, существуют многообразия, которые не допускают аналитически интегрируемых геодезических потоков, но имеют очень естественные потоки, интегриуемые в гладком смысле. Простейшим примером служат так назьваемые трехмерные SOL-многообразия. Геодезические потоки на них обладают еще одним удивительным свойством: они интегрируемы, но имеют положительную топологическую энтропию. В докладе также рассказано о классических и недавних результах о топологических препятствиях к интегрируемости (Динабург, Козлов, Тайманов, Патернайн) и способах построения интегрируемых геодезических потоков (Якоби, Клеро, Лиувилль, Штеккель, . . .).

24 февраля 2004 г. Заседание, посвященное реформе математического образования в средней школе.

По обсуждаемой проблеме выступили А. М. Абрамов, Д. В. Аносов и др.

Заседание 9 марта 2004 г. В. Ю. КАЛОшин. Линейная по времени неустойчивость в почти интегрируемых гамильтоновых системах (дополнение к теории Нехорошева).

Вопрос времени устойчивости гамильтоновых систем, близких к интегрируемым, является одним из важных в гамильтоновой динамике в связи с различными вопросами устойчивости, например, Солнечной системы. Если рассмотреть гамильтонову систему, $\varepsilon$-близкую к интегрируемой, $H_{\varepsilon}(x, I, t)$ в размерности $2 n+1$ (или с $n+1 / 2$ степенями свободы), то хорошо известная оценка Нехорошева времени устойчивости для $H_{\varepsilon}$ говорит, что для экспоненциалшно болшших времен,

$$
|t|<\exp \left(\varepsilon^{-a}\right)
$$

действие $I$ меняется не больше, чем на $\varepsilon^{b}$, где $a=b=1 / 2 n$. Однако зависимость показателей 
$a$ и $b$ от размерности становится важной для многомерных систем. Например, для $\varepsilon$, примерно равного $\exp (n)$, мы получим $\varepsilon^{a}=\varepsilon^{b}$ примерно равно 1 .

Приводится пример аналитического возмущения с $\varepsilon$, примерно равным $\exp (n)$, такого, что у $H_{\varepsilon}$ имеется траектория с линейным сносом действия: $|I(t)-I(0)|>c \varepsilon t$ для всех времен. Этот пример продолжает исследования Бургейна, Эрмана и других, которые показали, что выше указанное свойство может быть вьполнено для $t$, примерно равного $1 / \varepsilon$. Тем самым это показьвает, что по порядку оценка Нехорошева оптимална. Более того, в многомерных системах она не очень эффективна.

Заседание 16 марта 2004 г. А. Д. Брюно. Новое обобщение цепной дроби.

Алгоритм разложения числа в обычную цепную дробь обладает многими замечателшными свойствами. В том числе:

1. Он прост.

2. Он дает наилучшие рационалњные приближения к числу.

3. Он периодичен для квадратичных иррациональностей.

B XVIII, XIX и XX веках были сделаны многочисленные попытки обобщить этот алгоритм на векторы (Эйлер, Эрмит, Якоби, Дирихле, Пуанкаре, Гурвиц, Брун, Минковский, Клейн, Вороной, Перрон, Скубенко, Арнольд и др.). Но пока так и не найден алгоритм, обладающий свойствами 1 и 2 и свойством

$3^{\prime}$. Периодичность для кубических иррациональностей.

Только алгоритм Вороного обладает свойствами 2 и $3^{\prime}$, но он довольно громоздок. Многогранники Клейна-Скубенко-Арнолшда, хотя и дают геометрическую интерпретацию наилучших приближений, но не дают основы для хорошего алгоритма, что было выяснено в работах докладчика и В. И. Парусникова. Алгоритмические и геометрические концепции, заложенные в указанные обобщения, видимо, недостаточно отражали фундаментальные свойства цепной дроби.

В докладе предлагается новая двумерная концепция цепной дроби, которая затем обобщается на трехмерную ситуацию и позволяет построить алгоритм, обладающий свойствами 1,2 и $3^{\prime}$. Рассмотрены примеры с подробными вычислениями по новому алгоритму.

Заседание 30 марта 2004 г. Ю. С. Ильяшенко. О работах А.А. Болибруха в теории линейных дифференциальных уравнений.

Доклад можно было бы назвать “Три жемчужины теории комплексных линейных уравнений”. Рассказан пример Болибруха, доказьвающий неразрешимость проблемы Римана-Гилшберта в общем случае, теорема о разрешимости этой проблемы для неприводимой монодромии; достаточное условие приводимости системы с иррегулярной особой точкой к так назьваемой стандартной форме Бирхгофа (содержащей только лорановские, но не тейлоровские члены). Подробные доказательства этих результатов потребовали бы нескольких лекций. Докладчик попытался рассказать о соображениях, которые, по его мнению, представляли собой прорыв в решении перечисленных выше проблем и после которых далшнейшее решение оставалось, в основном, делом техники.

Заседание 6 апреля 2004 г. Б. М. ГУРЕвич. Термодинамический формализм и хаусдорфова размерность.

В конце 60-х годов 20-го века появился новый, “бесконечномерный” подход к задачам равновесной статистической физики, позволивший строго сформулировать и решить некоторые из этих задач, главньм образом - касающихся фазовых переходов. Этот подход оказал сильное воздействие на смежные области математики, прежде всего - на теорию динамических систем, в которой возникло новое направление, часто называемое термодинамическим формализмом.

В докладе рассказано о применении термодинамического формализма к вычислению хаусдорфовой размерности некоторых множеств, связанных с эргодической теоремой для динамических систем с многомерным временем.

Заседание 20 апреля 2004 г. А. С. ЧЕрный. Общая модель теории арбитража: вероятностный и возможностный подходы.

Доклад связан с финансовой математикой, более точно, с теорией арбитража. Это область финансовой математики, имеющая дело с нахождением справедливых цен производных финансовых инструментов. 
Доклад состоит из 5 частей:

1. Во введении кратко рассказано об основных задачах современной финансовой математики, о том, какое место в этой науке занимает теория арбитража, а также об основных идеях этой теории.

2. Кратко изложена теория арбитража на примере одношаговой модели с конечным числом активов.

3. Предложена общая модель теории арбитража, включающая в виде частных случаев большинство частных моделей.

4. Результаты, полученные в рамках общей модели, "спроецированы” на 2 конкретные модели: динамическую модель с конечным числом активов и одну статическую модель с бесконечным числом активов.

5. Предложен возможностньй подход к теории арбитража как альтернатива к обычному вероятностному подходу. 